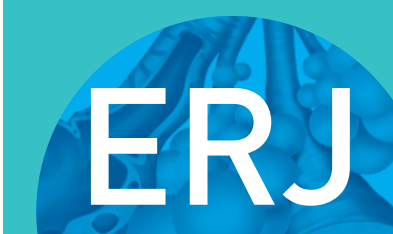

open research

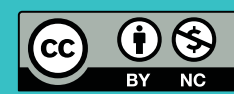

\section{Cost of illness of non-multidrug-resistant tuberculosis in Germany: an update}

\author{
Roland Diel ${ }^{1,2,3,4}$ and Albert Nienhaus ${ }^{4,5}$
}

Affiliations: ${ }^{1}$ Institute for Epidemiology, University Medical Hospital Schleswig-Holstein, Kiel, Germany. ${ }^{2}$ Lung Clinic Großhansdorf, Großhansdorf, Germany. ${ }^{3}$ Airway Disease Center North (ARCN), German Center for Lung Research (DZL), Großhansdorf, Germany. ${ }^{4}$ Institution for Statutory Accident Insurance and Prevention in the Health and Welfare Services (BGW), Hamburg, Germany. ${ }^{5}$ Institute for Health Service Research in Dermatology and Nursing, University Medical Center Hamburg-Eppendorf, Hamburg, Germany.

Correspondence: Roland Diel, Institute for Epidemiology, University Medical Hospital Schleswig-Holstein, Campus Kiel, Niemannsweg 11, Kiel, Schleswig-Holstein 24105, Germany. E-mail: roland.dieldepi.uni-kiel.de

\section{ABSTRACT}

Background: A total of 5429 new cases of tuberculosis (TB) were reported in Germany in 2018; out of the $3780 \mathrm{~TB}$ cases for whom drug susceptibility testing was available, the proportion of multidrug-resistant TB (MDR-TB) cases was only 3.1\% (118 cases).

Methods: On the basis of the current therapy guidelines of the German Central Committee against Tuberculosis, this study estimates the mean direct outpatient and combined in- and outpatient costs per non-MDR-TB patient from the perspective of the German statutory health insurance (SHI) system, together with costs arising from productivity losses and costs due to public health screening for TB in close contacts.

Results: From the insurance perspective, the mean outpatient costs (rounded) per case were $€ 1628$ for adults and $€ 1179$ for children for standard therapy; the mean cost of inpatient treatment amounted to $€ 8626$. The mean combined inpatient/outpatient cost was $€ 8756$ for adults and $€ 8512$ for children. As $95 \%$ of all TB patients were adults, the weighted treatment cost per patient in Germany in 2018 was $€ 8746$. These are in addition to the mean cost arising from productivity losses (€1839) and, weighted by pulmonary infectivity, cost of contact investigations ( $€ 368$ ), coming to a total of $€ 10953$.

Conclusion: Given the clear increase in the number of non-MDR-TB cases since 2015, TB is still a disease of significant economic impact in Germany.

@ERSpublications

Given the clear increase in the number of non-MDR-TB cases since 2015, TB is still a disease of significant economic impact in Germany https://bit.ly/30rkfvI

Cite this article as: Diel R, Nienhaus A. Cost of illness of non-multidrug-resistant tuberculosis in Germany: an update. ERJ Open Res 2020; 6: 00329-2020 [https://doi.org/10.1183/23120541.003292020].

This article has supplementary material available from openres.ersjournals.com

Received: 30 May 2020 | Accepted after revision: 13 July 2020

Copyright $\odot$ ERS 2020. This article is open access and distributed under the terms of the Creative Commons Attribution Non-Commercial Licence 4.0. 


\section{Introduction}

It has become a seemingly unavoidable convention that original papers on tuberculosis (TB) begin with a statement that the disease is still the world's most frequently encountered contagious disease, causing about 1.2 million deaths among HIV-negative people in 2018, with an estimated 10 million people contracting the disease that year, according to the World Health Organization (WHO) [1]. Although the socioeconomic problems associated with TB may vary considerably from country to country from a health economics point of view, it is necessary to update and methodically validate cost calculations of the various facets of TB disease as a prerequisite to managing healthcare activities to counter TB as effectively as possible, including "low-burden" countries.

The most recent cost calculation available in Germany for a "standard" TB patient was published in 2012 [2], mainly on the basis of German cost data from 2009, followed 2 years later (in 2014) by a burden of disease analysis of TB in the European Union [3]. Since then, the official number of new cases of TB in Germany reported to the Robert Koch Institute has increased from 4444 reported cases in 2009 [4] to a total number of 5429 cases in 2018, mainly due to an ongoing stream of refugees since 2015 [5]. Whereas in $2009,44.6 \%$ of TB cases were reported among immigrants [4], the proportion of foreign-born cases in 2018 - as in the previous 2 years - accounted for almost three-quarters of all patients [5]. As 8 years have lapsed since the publication of our work, an up-to-date and more comprehensive cost-of-illness study is overdue. Given its aim to provide a realistic report of the current morbidity costs for non-multidrug-resistant TB (non-MDR-TB) in Germany, this update on the subject extends the scope to encompass a societal point of view and include all types of non-MDR-TB, not only those affecting the lungs. An update of the 2014 cost analysis of MDR-TB cases in Germany [6], the proportion of which increased to $3.1 \%$ (118 cases) in 2018 [5], requires a different approach than that for non-MDR-TB. The former must be based on complex susceptibility profiles of the single MDR-TB strains and will therefore be published separately.

\section{Methods}

This analysis only considers costs that must be covered by law or are attributable to standard modern therapy procedures. Because they cannot be adequately modelled, some of the costs that may arise from cases of $\mathrm{TB}$ are not taken into consideration, e.g., current data that reflected costs incurred for rehabilitation, which were covered by the German pension insurance and previously included [2], are no longer available. It is also not possible at the current time to assess the total cost of isoniazid $(\mathrm{H})$ preventive therapy (PT) in persons exposed to infectious TB patients, as latent tuberculosis infection (LTBI) and subsequently performed PT is exempted from notification to the German public health authorities. Therefore, although such therapy is paid for by German statutory health insurance (SHI), no cost data on the number and duration of actually completed PT courses are available to date.

To adopt a societal point of view, the following cost categories are defined and calculated, where possible based on the most recent available data in each case:

1. Costs for diagnosis and therapy of $\mathrm{TB}$ as recommended by the current guidelines of the German Central Committee against Tuberculosis (Deutsches Zentralkomitee zur Bekämpfung der Tuberkulose, DZK) [7] and covered by the SHI system, categorised into inpatient and outpatient sectors.

2. Indirect costs incurred by the general economy arising from loss of productivity.

3. Costs incurred by public health units for the execution of contact investigations as required by the Federal Law on Infectious Diseases (IfSG).

Our calculations of the direct costs are based on the rates established by the Uniform Appraisal Scale (EBM, Einheitlicher Bewertungsmaßstab) [8] and the Institute for the Hospital Remuneration System (InEK, Institut für das Entgeltsystem im Krankenhaus) data [9, 10] for the German diagnosis related groups (DRG) system; these constitute the costs effectively incurred by the SHI. Specifically, we used data provided by the German Hospital Federation as the basis for an evaluation of daily reimbursement costs to the SHI for TB patients hospitalised for more than 14 days.

Because no representative random sampling study of the number of workdays lost due to TB in Germany (ICD-10 A15-A19) exists, indirect costs are calculated in a simplified fashion by taking the latest available sick leave period (in 2018) for gainfully employed persons insured under mandatory statutory schemes by the statutory health insurance group AOK (Allgemeine Ortskrankenkasse), the SHI fund with the largest number of members in Germany. It is not possible to say how representative the AOK patient contingent is for all people insured under SHI. However, given AOK's large market share throughout Germany, the probable deviations are likely to be negligible. Loss of productivity from the point of view of the general economy is then calculated on the basis of the employment rate in the country's total population.

To estimate the costs incurred by public health units for executing contact investigations as required by the Federal Law on Infectious Diseases (IfSG), we analysed the latest available performance data collected in 
the centralised TB unit of Hamburg, a metropolitan region that in the past 5 years has had the highest cumulative TB incidence of all federal states in Germany (median 6.4 per 100000 inhabitants [5]).

\section{Epidemiological background}

Since the last German cost-of-burden analysis for TB [2] was published, the epidemiological data and cost figures have both changed remarkably.

In Germany in 2018, the Robert Koch Institute in Berlin was notified of a total of 5429 TB cases, resulting in a TB incidence rate of 6.5 cases per 100000 population [4]. Only 215 new cases were reported in children, meaning that the large majority of cases, 5212 or $96 \%$, relate to adults (aged 15 years or older). Treatment was provided in hospital wards in $89.5 \%$ of the TB cases, without any significant differences with respect to sex, age and origin [4].

With a total of 5429 TB cases, of which 5427 cases featured a recorded age, $72.1 \%$ of the cases $(n=3914)$ were pulmonary TB, of which 163 were in children and 3751 in adults. This means that 163 of 215 cases or $75.8 \%$ related to pulmonary TB in children while 3751 of 5212 cases or $72.0 \%$ related to pulmonary TB in adults. Seventy-nine out of the 163 children with pulmonary TB were culture-positive (48.5\%), against 2980 out of the 3751 adults (79.4\%) (personal communication; W. Haas, Robert Koch Institute, Berlin, Germany). In total, $78.2 \%$ of all pulmonary $\mathrm{TB}$ cases were culture-positive. Of the remaining non-pulmonary $\mathrm{TB}$ cases, $40.8 \%$ of the children and $64.0 \%$ of the adults were culture-positive.

\section{Diagnosis and therapy}

The diagnostic algorithm for TB is largely defined by the EBM catalogue of procedures for which the SHI provides coverage. Currently, these may begin with a Mendel-Mantoux tuberculin skin test to verify infection, followed by a more specific interferon-gamma release assay (IGRA). In patients with suspected pulmonary TB this is generally followed by a chest X-ray (affected organ) and bacteriological confirmation - usually from sputum or bronchoalveolar lavage following referral to a pneumonologist, with microscopic verification of acid-fast bacillus (indicator of contagiousness) and/or culture (pathogen verification) together with a sensitivity test of the first isolate for anti-TB chemotherapy drugs. In any case of suspected $\mathrm{TB}$, nucleic acid amplification (NAAT) assays should in addition be performed routinely for rapid identification of the M. tuberculosis complex or for MDR, e.g. by the WHO-endorsed Xpert MTB/RIF Ultra $^{\oplus}$ (Cepheid), detecting R-resistance in sputum samples and other body fluids, especially in patients from MDR high-burden countries and in patients with known previous contact to MDR-TB cases.

According to the guidelines of the DZK [6], the therapy for pulmonary TB and also for all extrapulmonary varieties of TB disease (apart from TB of the central nervous system) lasts at least 6 months, a regimen that keeps recurrence rates to a minimum (3\%). In view of the fact that in Germany $13.0 \%$ of all culturally-verified cases of TB are resistant to at least one of the first-line drugs, the quadruple combination with isoniazid $(\mathrm{H})$, rifampicin $(\mathrm{R})$, pyrazinamide $(\mathrm{Z})$ and ethambutol $(\mathrm{E})$ is recommended. After an "initial phase" of 2 months that precedes the arrival of the resistance results, patients with fully sensitive strains are then treated with $\mathrm{H}$ and $\mathrm{R}$ for a further 4 months in the subsequent continuity phase. In children only, an initial phase with three drugs $(\mathrm{H}, \mathrm{R}, \mathrm{Z})$ is necessary if there are no risk factors for resistance (prior treatment, etc.). This combination can be used because colonisation in children is relatively limited and consequently the probability of spontaneous resistance mutations is very low. The occurrence of drug-resistant TB in children (19 children in 2018 [5]) remains an exception in Germany.

\section{Basic assumptions for calculating outpatient costs}

1) The responsibility for management of the diagnosis and therapy for primary outpatient treatment or secondary outpatient treatment following initial hospitalisation lies with the general practitioner throughout the course of the disease. For the purposes of simplification, the general practitioner will either refer the patient directly to the hospital where the patient will start his/her diagnostic procedures and primary therapy as an inpatient, or alternatively to the resident lung specialist who will care for the patient primarily as an outpatient or, if the patient is initially treated as an inpatient, provide further outpatient care. Where there were fee schedule positions for different age groups, the most probable position in each case (younger adults) where TB disease is most prevalent is applied. Because in Germany non-pulmonary adult TB patients are also mostly treated by pulmonologists, the cost figures for treating pulmonary TB are applied to treatment of non-pulmonary TB cases - with the exception of radiological monitoring and bronchoscopy.

2) The drug costs are calculated on the basis of the recommended maximum dose for the drugs being administered on a daily basis. The cheapest drugs with the corresponding effective substance are used, according to the 2019 issue of the LAUER-TAXE ${ }^{\circledR}$ Online 4.0 (German drugs directory including the mandatory discounts that pharmaceutical manufacturers and pharmacies are required to apply in favour 
of the SHI; last access: 1 December 2019). The quoted costs have had the legally prescribed discounts in favour of SHI deducted. These are defined by paragraphs 130 and 130a of the German Social Insurance Code (Fünftes Buch Sozialgesetzbuch; SGB V) and include the pharmacy discount of $€ 1.77$ pursuant to $\$ 130$ SGB V, the manufacturer discount pursuant to $\$ 130 \mathrm{a}$ (1) SGB V, the manufacturer discount pursuant to $\$ 130 \mathrm{a}$ (3b) SGB V for patent-free drugs with the same effect and, if applicable, the discount according to $\$ 130$ a (3a) SGB V.

3) According to the German guidelines [6], quadruple therapy including $\mathrm{E}$ is also used in adults for culturally unconfirmed TB.

4) Although numerous side effects can occur throughout therapy, costs for adjuvant medication do not apply because data on treating and monitoring these side effects is not routinely collected.

5) As the rate of hospitalisation (89.5\%) reported for German TB patients in [4] did not differentiate between any sites of TB, this figure was assumed for patients with both pulmonary and non-pulmonary TB.

6) Costs of bronchoscopy were included for all patients with pulmonary TB, irrespective of whether the patients' samples were eventually culturally positive or negative. Costs of bronchoscopy were not included for patients with non-pulmonary TB or children.

7) This calculation adds all attributable costs of state-of-the-art diagnostics and therapy in children and adults as recommended by relevant guidelines/recommendations utilising proportions from routine surveillance data and fixed or pre-weighted costs. It does not take into consideration outcomes from clinical trials or different size samples. Confidence intervals are not provided, as their application to this non-probabilistic model would have been inappropriate.

\section{Diagnosis and monitoring of outpatients and patients following initial hospitalisation}

The monitoring of anti-TB therapy described below is closely related: three expectorated sputum samples (for the larger yield) are collected on separate days for microscopy in patients with suspected pulmonary TB in the initial phase and then every 4 weeks until negative results are obtained, i.e. at least five times for a presumed conversion within 8 weeks. Cultures are bred in the initial phase (positive culture results followed by resistance test), then after 4 and 8 weeks (conversion should have occurred by that time) and then once more towards the end of the therapy (verification of successful therapy according to WHO criteria). For patients with non-pulmonary TB it was assumed that only one culture would be performed at baseline.

1) The first positive culture in the initial phase is subjected to resistance testing. For simultaneous rapid TB diagnosis, a NAAT can also be used in microscopically negative samples and molecular fast resistance testing performed in addition in cases of positive NAAT or microscopy.

2) Material costs of the tuberculin skin test (TST) (PPD Mantoux). The TST licensed for Germany is PPD RT 23, 2 TU $0.1 \mathrm{~mL}$ from the Danish Statens Serum Institute, and distributed by Pharmore Ltd. The material costs per TST (glass vials) are classified as practice supplies and may not be charged separately according to the German EBM system.

3) Since 2019 an IGRA test can be charged for in an outpatient context following a TST, even if the TST is negative but the patient is suspected of having TB. We assumed that not only a TST but also a subsequent IGRA will generally also be performed on patients treated solely in an outpatient context.

4) Blood work: in accordance with the DZK guidelines, a broad blood profile is necessary before therapy starts, together with definition of kidney retention values (creatinine, urea), liver values (GOT, GPT, bilirubin, GGT [which is required as an alcohol abuse parameter and also allows for differentiation between $\mathrm{H}$-induced hepatitis and $\mathrm{R}$-induced cholangitis in case this value increases as a result of the medication]); it is also advisable to determine uric acid level in adults (as a compliance parameter because an increase under $\mathrm{Z}$ is almost certain), together with hepatitis serology (HBs-Ag, Anti-HBc), as well as HIV serology. Liver values are controlled 2-4 weeks after starting the therapy, and every 4 weeks thereafter (bilirubin is not checked if there are no anomalies in the liver parameters in the first test and subsequent checks). Uric acid values are checked only every 4 weeks until $\mathrm{Z}$ is stopped, i.e. altogether only twice in standard therapy if prior hyperuricemia was not reported. Blood tests and renal retention parameters are checked every month through to the end of the therapy, together with checks of the liver values.

5) Ophthalmic examination: under ethambutol (E), before therapy starts and usually every 4 weeks.

6) Chest X-rays (given pulmonary TB): in the initial phase, after 4 weeks (to control whether the TB is reacting to the therapy), and after 8 weeks (end of the initial therapy: success assessment). Then X-ray check-ups in the 4th and 6th months are sufficient, followed by check-ups 6, 12 and 24 months after the end of treatment. For patients with non-pulmonary TB, only one X-ray examination at baseline is considered necessary for the exclusion of pulmonary TB. Computed tomography or ultrasound examinations are not routine procedures for diagnostic evaluation or periodic follow-up in the 
outpatient setting. If deemed necessary, these will most frequently be conducted in the hospital. As such, the corresponding costs are already covered by the DRG per-case reimbursement and will not be enumerated separately.

\section{Results}

Inpatient cost (direct hospital costs)

In relation to inpatients, hospital costs have been based on the uniform German G-DRG system since 1 January 2004, which allocates each case to a diagnosis-related group. Under this system, reimbursement of hospital services is no longer based on fixed daily rates for the period of stay, but focuses on the nature and severity of the diseases. A calculated base rate for the hospitals in the corresponding federal state is then multiplied by the specific cost weight of a disease, resulting in the proceeds for the hospital providing the treatment. For TB, there are three categories; while the costs for E76B cases (with severe complications, hospital stay less than 14 days) and E76C cases (without severe complications, hospital stay less than 14 days) are set by multiplying the national base rate ("Bundesbasisfallwert") for 2019 of $€ 3544.97$ by different cost weights depending on their different degree of disease severity (1.097 for E76B and 0.937 for E76C), the costs per day for patients being diagnosed and treated in hospital for longer than 14 days (E76A, introduced in 2007) have to be negotiated separately between third-party payers and the respective hospital and may vary accordingly.

Based on a calculated mean length of hospital stay of 25 (25.14) days per patient and a per-day reimbursement of $€ 320.77$ (personal communication; C. Jaeger, Deutsche Krankenhausgesellschaft e. V., Berlin, Germany) for patients hospitalised for more than 14 days, the mean hospitalisation costs per non-MDR-TB patients are $€ 8625.60$ (details of derivations are provided in the Online Supplement).

\section{Outpatient costs}

Outpatient costs at the expense of the SHI comprise costs of first-line drugs for 6 months (for primary outpatients) or 6 months minus 25 days (for patients following hospitalisation) according to the 2019 issue of the LAUER-TAXE ${ }^{\circledast}$ and the costs of services and providers for diagnosing and monitoring as suggested above, according to the EBM scheme revised in 2019 [8], respectively. Costs of first-line drugs are presented separately for primary outpatients and outpatients following primary hospitalisation in tables 1-3. Table 4 shows the billable fee schedule positions that have to be adapted to the different scenarios.

The weighted outpatient costs for adults with pulmonary and non-pulmonary TB are $€ 1628.21$ and the outpatient costs for those adults after the hospital stay are €966.31. The corresponding costs for children are $€ 1179.05$ and $€ 747.10$, respectively (see Online Supplement).

\section{TABLE 1 List of medical drugs for non-MDR-TB treatment available in Germany}

\begin{tabular}{|c|c|c|c|c|}
\hline Name & Abbreviation & $\begin{array}{l}\text { Price after subtracting } \\
\text { legally required } \\
\text { discounts/price per } \\
\text { packet }\end{array}$ & $\begin{array}{l}\text { Form/pharmacy } \\
\text { retail price }\end{array}$ & $\begin{array}{l}\text { Price of daily } \\
\text { defined dose }\end{array}$ \\
\hline $\begin{array}{l}\text { Rifampicin } 600 \mathrm{mg} \\
\text { EREMFAT }\end{array}$ & RIF & $€ 258.96\left[€ 1.77^{\#} ; € 14.64^{\text {T] }}\right]$ & $\begin{array}{l}100 \text { film-coated } \\
\text { tablets: } € 275.37\end{array}$ & $1 \times 1: € 2.59$ \\
\hline $\begin{array}{l}\text { Ethambutol } \\
\text { (EMB-FATOL) } \\
\mathbf{4 0 0 ~} \mathrm{mg}\end{array}$ & $E$ & $€ 38.71\left[€ 1.77^{\#} ; € 1.73^{\text {ๆ1 }}\right]$ & $\begin{array}{l}100 \text { film-coated } \\
\text { tablets: } € 42.21\end{array}$ & $\begin{array}{l}4 \times 1: € 1.55 \\
\quad\left(15 \mathrm{mg} \cdot \mathrm{kg}^{-1}\right)\end{array}$ \\
\hline $\begin{array}{r}\text { Isoniazid ISOZID } \\
\text { comp. } 300 \mathrm{mg}\end{array}$ & $\mathrm{H}$ & $€ 33.98\left[€ 1.77^{\#} ; 6.64^{\text {91 }}\right]$ & $\begin{array}{l}100 \text { film-coated } \\
\text { tablets: } € 42.39\end{array}$ & $1 \times 1: € 0.34$ \\
\hline $\begin{array}{l}\text { Pyrazinamide } \\
\text { JENAPHARM } \\
500 \mathrm{mg}\end{array}$ & Z & $€ 31.23\left[€ 1.77^{\#} ; €-\right]$ & 100 tablets: $€ 33.00$ & $\begin{array}{l}4 \times 1: € 1.25 \\
\text { [2000 mg in } \\
\text { children] } \\
5 \times 1: € 1.56 \\
\text { [2500 mg in } \\
\text { adults] }\end{array}$ \\
\hline
\end{tabular}




\begin{tabular}{|c|c|c|c|}
\hline Medication" & Costs/day (rounded) & Costs ${ }^{\pi}$ & Costs post-hospital stay (minus 25 days) \\
\hline Rifampicin $^{+}$ & $€ 2.59$ & $€ 466.20$ & $€ 401.45$ (155 days) \\
\hline Isoniazid $\$$ & $€ 0.34$ & $€ 61.20$ & $€ 52.70$ (155 days) \\
\hline Ethambutol ${ }^{f}$ & $€ 1.55$ & $€ 93.00$ & $€ 54.25$ (35 days) \\
\hline Pyrazinamide $\# \#$ & $€ 1.56$ & $€ 93.60$ & $€ 54.60$ (35 days) \\
\hline- & - & $€ 714.00$ & $€ 563.00$ \\
\hline
\end{tabular}

\#: Based always on the smallest pack available for the necessary minimum period of treatment. The daily therapy costs are calculated from the quotient between the intake quantity stated in the dosing instructions and the pack quantity and multiplied by the number of treatment days. ": Based on an average treatment period of 180 days for isoniazid and rifampicin; 60 days are taken for pyrazinamide, ethambutol and streptomycin using the recommended maximum dose in each case. ${ }^{+}:$Eremfat $600 \mathrm{Tbl}$, one tablet taken once a day as instructed (maximum dose $600 \mathrm{mg}$ ). ${ }^{\S}$ : ISOZID comp $300 \mathrm{Tbl}$, one tablet taken once a day as instructed (maximum dose $300 \mathrm{mg}$ ). ${ }^{f}$ : EMB FATOL 400 Tbl., four tablets taken once a day (maximum dose $1600 \mathrm{mg}$ ). \#\#: Pyrazinamide JENAPHARM 500 Tbl., five tablets taken once a day (maximum dose $2500 \mathrm{mg}$ ).

\section{Combined inpatient/outpatient TB costs}

Hospital treatment was provided in $89.5 \%$ of all TB cases, so in adults the weighted costs for a standard TB case are $[(€ 8625.60+€ 966.31) \times 0.895]$ plus $(€ 1628.21 \times 0.105)$, i.e. $€ 8755.72$.

For children, the combined costs can be calculated as follows: $(€ 8625.60+€ 747.10) \times 0.895$ plus $(€ 1179.05 \times 0.105)$, i.e. $€ 8512.37$. As $96 \%$ of all TB cases related to adults, the average costs for diagnosis and treatment in Germany in 2018 per patient were $(€ 8755.72 \times 0.96)$ plus $(€ 8512.37 \times 0.04)$, i.e. $€ 8745.99$. Table 5 provides the calculations for the different cost components and the total treatment costs from the perspective of the SHI.

\section{Indirect costs (loss of productivity)}

From a societal point of view, losses in productivity arising from disease, referred to as "indirect costs", must be included in disease cost estimates. Following the human capital approach, indirect costs represent the production loss for the economy at large caused by absence from the workplace on sick leave. According to the "Hanoverian Consensus" [11], the evaluation of productivity losses caused by sickness should disregard differences between work sectors, age or sex ("all employees"), with the average gross income for the period from non-self-employed employment.

The average productivity loss to cover the self-employed as well is calculated as follows: productivity loss = sick leave days $\times$ [gross income from non-self-employed work divided by the number of employer-dependent gainfully employed times 365 days]. As more detailed information is not available, an estimate is recommended on the basis of the data provided by the Federal Statistics Department; the mean sick leave duration in 2018 of members of the AOK (previously mentioned) insured under mandatory

TABLE 3 Statutory health insurance costs for outpatient therapy in children: medication

\begin{tabular}{|c|c|c|c|}
\hline Medication" & Costs/day (rounded up) & Costs" & Costs post-hospital stay \\
\hline Rifampicin $^{+}$ & $€ 2.59$ & $€ 466.20$ & $€ 401.45$ (155 days) \\
\hline Isoniazid ${ }^{\S}$ & $€ 0.34$ & $€ 61.20$ & $€ 52.70$ (155 days) \\
\hline Pyrazinamide ${ }^{f}$ & $€ 1.25$ & $€ 75.00$ & $€ 43.75$ (35 days) \\
\hline - & - & $€ 602.40$ & $€ 497.90$ \\
\hline \multicolumn{4}{|c|}{$\begin{array}{l}\text { \#: Based always on the smallest pack available for the necessary minimum period of treatment. The daily } \\
\text { therapy costs are calculated from the quotient between the intake quantity stated in the dosing instructions } \\
\text { and the pack quantity and multiplied by the number of treatment days. ๆ: Based on an average treatment } \\
\text { period of } 180 \text { days for isoniazid and rifampicin, and } 60 \text { days for pyrazinamide using the recommended } \\
\text { maximum dose in each case. }{ }^{+} \text {: Eremfat } 600 \text { Tbl., one tablet taken once a day as instructed (maximum dose } \\
600 \mathrm{mg} \text { ). } \S \text { : ISOZID comp } 300 \text { Tbl., one tablet taken once a day as instructed (maximal dose } 300 \mathrm{mg} \text { ) } \\
\text { f: Pyrazinamide } 500 \text { Tbl., four tablets taken once a day as instructed (in children maximal dose } 2 \mathrm{~g} \text { ). }\end{array}$} \\
\hline
\end{tabular}


TABLE 4 Statutory health insurance costs of diagnosing and monitoring pulmonary TB in the outpatient setting

\section{Medical services}

\section{General practitioner}

1. Flat rate coverage; irrespective of number of visits by patients per quarter (GOP 03000)

Alternatively:

2. Paediatric flat rate GOP 04000 lage 0 to 4 )

3. Detailed conversation (GOP 04230); can also be charged if the discussion is only with caregivers without the child's presence)

4. Retainer fee (general practitioner), once per quarter (GOP 03040); identical to the paediatric billing code GOP 04040

\section{Pneumological diagnostics}

5. Pneumological consultation; 6 to 59 years of age (GOP 13641)

6. Surcharge (pneumologist), once per quarter (GOP 13644)

7. Bronchoscopy (GOP 13662)

8. Pneumologist BAL (GOP 13663), additional fee on GOP 13662

\section{Methods}

9. Mantoux TST (GOP 02200)

10. IGRA testing (GOP 50112 following GOP 0220)

11. ECG (GOP 27320); cannot be charged separately

12. X-ray in two planes (GOP 34241); a consultation cannot be charged

13. For ethambutol treatment: ophthalmologic consultation (GOP 06211 for insured patients aged between 6 and 59 years)

\section{Microbiology}

14. Microscopy test for mycobacteria (GOP 32176 ) laspiration of gastric juice via tube in children cannot be charged separately)

15. NAAT (GOP 32825)

16. Culture test for mycobacteria (GOP 32747 ) per material

17. Differentiation of mycobacteria (GOP32764) if positive

18. Molecular fast resistance testing (GOP 50110 if NAAT or microscopy is positivel

19. Resistance definition GOP 32770) per mycobacteria type

\section{Laboratory investigation}

20. HIV serology, immune-assay combination test (GOP 32575)

21. Anti-HBc (GOP 32 614)

22. HBs-Ag (GOP 32781)

23. Chloride (GOP 32084)

24. Sodium (GOP 32083)

25. Potassium (GOP 32081 )

26. Calcium (GOP 32082)

27. Creatinine, Jaffe method (GOP 32066 )

28. Uric acid (GOP 32964)

29. Urea (GOP 32065)

30. Blood count (GOP 32122)

31. Bilirubin total (GOP 32058)

32. Gamma-glutamyl transferase (GOP 32071)

33. Glutamate-oxaloacetate transaminase (GOP 32069)

34. Glutamate-pyruvate transaminase (GOP 32070)
Individual

payment $€$

16.99

25.54

9.74

15.58

21.86

4.44

106.93

26.19

0.97

58

16.45

13.69

5.20

61.40

34.90

28.40

82.03

39.50

4.45

5.90

5.50

0.25

0.25

0.25

0.25

0.25

0.25

0.25

1.10

0.25

0.25

0.25

0.25
Frequency

5 [quarters]

5 [quarters]

2

5 [quarters]

77.90

109.30

22.20

106.93

26.19

0.97

58

131.60

13.69 lonly be charged as flat once per quarter]

5 (3 if closed TB)

1
4 (1 if closed
TB)

1 ( 0 if closed TB)

1 (0 if closed TB)

1 (0 if closed TB)

1

amplification test. 
TABLE 5 Age-dependent direct treatment (statutory health insurance) costs for non-MDR-TB

\begin{tabular}{lcc} 
Age group & $\mathbf{0}$ to $<15$ years & $\geqslant 15$ years \\
\hline Hospital treatment & & $89.5 \%$ \\
Mean hospitalisation rate & $89.5 \%$ & $€ 8625.60$ \\
Mean reimbursement per case & $€ 8625.60$ & $79.4 \%$ \\
Culture confirmation & & $64.0 \%$ \\
Pulmonary TB & $48.5 \%$ & 25.14 \\
Non-pulmonary TB & $40.8 \%$ & $€ 1732.69$ \\
Mean hospital stay days & 25.14 & $€ 1359.54$ \\
Primary outpatient therapy ladults) & - & $€ 1628.21$ \\
Pulmonary TB & - & $€ 1020.69$ \\
Non-pulmonary TB & & $€ 826.47$ \\
Weighted average & - & $€ 966.31$ \\
Post-hospital outpatient therapy ladults) & - & - \\
Pulmonary TB & & - \\
Non-pulmonary & & \\
Weighted average & $€ 1225.74$ & - \\
Primary outpatient triple therapy (children) & $€ 1033.82$ & \\
Pulmonary TB & $€ 1179.05$ & - \\
Non-pulmonary TB & & - \\
Weighted average & $€ 785.10$ & \\
Post-hospital outpatient triple therapy (children) & $€ 628.05$ & \\
Pulmonary TB & $€ 747.10$ & \\
Non-pulmonary TB & - & \\
Weighted average & $€ 8512.37$ & \\
Combined inpatient/outpatient costs & & \\
Standard TB adults & & \\
Standard TB children & & \\
Weighted average & &
\end{tabular}

statutory insurance schemes throughout Germany for TB (ICD-10 A15-A19) was 73641 days/2537 cases, i.e. 29.0 days per case (personal communication; M. Meyer, Wissenschaftliches Institut der AOK, Berlin, Germany). For 2018, the loss of productivity in Germany is considered to be $€ 119.37$ per sick leave day [12].

Accordingly, the loss of productivity per person ill with TB could be calculated at €3461.73 $(€ 119.37 \times 29.0$ days). To allow for a general statement applicable to all TB patients (both employed and unemployed), this figure firstly has to be weighted by multiplying it by the gross employment rate of $53.13 \%$, i.e. the proportion of employees relative to the overall population in Germany (43360000/81613000 [13]). This means that the weighted mean of productivity loss of all TB patients is $€ 1839.22$.

\section{Costs of public health contact investigations}

According to $\$ 16$ and $\$ 25$ of the Federal Law on Infectious Diseases (IfSG), responsibility for diagnosis of LTBI before sending a screened contact to a pulmonology practice lies with the public health service. The public health departments to whom each patient suffering from active TB disease has to be reported will routinely perform contact investigations according to $\$ 16$ and $\$ 25$ of IfSG. Thus, each case of a person with infectious, i.e. culture-confirmed pulmonary TB disease (the "index case"), results in further costs for those contact investigations, the purpose of which is to minimise possible chains of infection developing due to secondary TB cases of diseased contacts. A further exercise is "source tracing" in patients with TB that may have developed very fast, such as tuberculous pleuritis or meningitis, and/or in children.

Contact persons aged at least 15 years (and optionally children aged between 5 and 14 years) are tested solely by an IGRA to detect recent MTB infection caused by the infectious index case. When testing children aged below 5 years, the Mantoux TST is the method of choice. Due to a loss of experience in performing the TST, it is often no longer performed in the health authorities themselves but in commissioned children's hospitals or by paediatricians in private practice where reimbursement follows the EBM.

According to the current guidelines of the German Central Committee against Tuberculosis [14], a contact person with a positive test has to undergo a chest X-ray in order to exclude a hitherto unknown active TB which may be asymptomatic. A second chest X-ray examination 1 year later is mandatory, with or without 


$\begin{array}{ll}\text { TABLE } 6 \text { Costs of contact investigations performed by the Public Health Office } & \text { Costs } \\ & € 73.07 \\ \text { Costs of chest X-ray procedure performed in a public health institution } & € 35.00 \\ \text { Laboratory costs of performing the QuantiFERON-TB Gold in Tube test (QFT) on behalf of the } & \\ \quad \text { Public Health Office } & € 11.02 \\ \text { Costs of performing the QFT by Public Health Office } & € 26.75 \\ \text { Costs of counselling a test positive contact on PT } & € 0.43 \\ \text { Costs of informing a pulmonologist before starting PT (weighted with the absolute number } & \\ \text { of contacts) } & € 31.90 \\ \text { Administrative costs of sending children }<5 \text { yrs to contact investigations in instructed } & \\ \quad \text { hospitals or surgeries per child } & € 69.36 \\ \text { Screening costs per child }<5 \text { yrs in instructed hospitals or paediatric practices, no LTBI } & € 84.37 \\ \text { Screening costs per child }<5 \text { yrs in instructed hospitals or surgeries, LTBI } \\ \text { Weighted screening costs per child }<5 \text { yrs (10.9\% TST positive), administrative costs excluded } & € 71.03 \\ \text { Weighted screening costs per child }<5 \text { yrs, administrative costs included } & € 102.93\end{array}$

preceding preventive treatment, to avoid overlooking an active TB disease that may have spontaneously developed in the meantime or during the isoniazid PT. Based on statistics of the Hamburg-Central Public Health Department, the calculated cost components for covering the different tasks are shown in table 6, resulting in a total of $€ 368.23$ per TB case (for details see Online Supplement).

\section{Total costs per non-MDR-TB case from a societal perspective}

Taking all costs together, the mean costs per non-MDR-TB case in Germany is $€ 8746$ (rounded treatment costs) plus $€ 1839$ (loss of productivity) and $€ 368$ (public health investigations), adding up to a total of $€ 10953$.

\section{Discussion}

Full transparency on the current costs of TB disease in European low-burden countries is a prerequisite for strengthening resource-stratified approaches to preventive interventions, e.g. immigrant screening. Healthcare systems in Europe not only differ substantially, but also undergo constant change in terms of how hospitals are reimbursed, experiencing unexpected epidemiological challenges and being bound by new treatment guidelines. Given that the most recently available cost calculation for non-MDR-TB in Germany, published in 2012 and partially utilising cost data from 2009 [2], could be considered outdated, we also took the opportunity to update the cost data of contact investigations by the local public health offices, the calculation of which was delayed for even longer [15].

Surprisingly, since 2012 only a few studies on the costs of non-MDR-TB have been performed in other European countries, and even less information has been provided in those on the single components of aggregated costs: De VRIES et al. [16], in their complete country-wide review analysis which included 1138 patients with non-MDR-TB and 20 with MDR/XDR-TB, estimated the per-patient treatment costs of non-MDR-TB at $€ 7850$, but did not address indirect costs or other cost components. FloE et al. [17] calculated the excess health-related costs of Danish TB patients and their spouses between 1998 and 2010 by matching 8433 patients with 337070 controls and 3485 spouses with 17403 controls, but did not include costs related to contact investigation procedures. Finally, Gullón et al. [18], in their prospective study on 312 patients in Spain collected between September 2014 and September 2015, estimated the average cost per TB case at $€ 10262.62$ but also included MDR-TB cases and assumed that the 2012 figure of $€ 921.80$ provided by Diel et al. [2] as an estimate for the per-patient cost of contact tracing would also apply to Spain, without performing their own calculations.

When comparing the mean combined inpatient/outpatient costs for non-MDR-TB of our previous study and adjusting the previous figure of $€ 7361.97$ to the 2019 prices of our update for inflation, based on an inflation rate since that time of $10.21 \%$ (www.finanzen-rechner.net/inflationsrechner.php), the calculated treatment costs covered by the SHI have only increased by a total of $7.8 \%$ (€8746 instead of the inflation-based figure of $€ 8114$ ). This is mainly due to the $17.2 \%$ increase in hospitalisations from $70.1 \%$ in 2009 , the starting point of our previous analysis, to $89.5 \%$ in 2018 . However, given hospital treatment, the length of hospitalisation has significantly fallen since 2009, from a mean of 30 days to 25 days, thus mitigating further cost increases. 
Regarding productivity losses, the average number of days off work due to TB disease in our update was also lowered by nearly 5 days (29.0 instead of 33.7 days), resulting in lower indirect costs of $€ 710$ per TB patient ( $€ 1839$ instead of the inflation-adjusted figure of $€ 2549)$. With respect to contact investigations, either performed by or on behalf of and paid for by public health services, new calculations were necessary. New guidelines of the German Central Committee [16], which came into effect in 2012, narrowed the selection of contacts of infectious TB patients to be investigated to close contact persons only and replaced Mantoux testing by IGRA in persons aged at least 15 years, thus avoiding a second visit of the contact individual to read a TST after 48 to $72 \mathrm{~h}$. Accordingly, on average, only 7.5 contacts per infectious TB case were screened in $2017 / 2018$, in contrast to 12 contacts intended to be screened in the previous analysis, thus resulting in significantly lower costs of about $€ 372$ per TB case instead of the previous inflation-adjusted estimate of $€ 1016$.

Omitting the costs of rehabilitation, which could not be included in the update because no current data were available, the total calculated costs per TB patient from the societal perspective in $2019, € 10953$, are even slightly lower than the 2011 costs of $€ 11679$ (when adjusted for inflation).

Our study has several limitations. Although the study provides a bottom-up analysis of the costs incurred in diagnosing and treating a typical case of TB disease in Germany, it probably underestimates the real costs of non-MDR-TB. Some patients may have resistance or intolerance to any of the four first-line drugs, especially isoniazid. As it is recommended in such cases to administer a fluoroquinolone in the initial period and/or to extend the continuation period [7], additional costs may be incurred for those patients. However, as in our previous analysis, such discretionary acts were not taken into consideration, as neither data on the actual prolongation of the short course treatment nor data on the chosen alternative regimens are available. Some additional procedures such as computer tomography or ultrasound examinations, which may above all play a role in diagnosing and monitoring of extrapulmonary $\mathrm{TB}$, have not been considered in our cost calculation. However, as these examinations are more likely to be conducted in hospitals than in outpatient settings, they will usually fall under the DRG reimbursement that covers all examinations during a TB patient's hospital stay.

Furthermore, costs arising for the public health system due to contact investigations could not be fully covered in our calculation, e.g. the costs arising for social workers whose predominant task is to identify close contact individuals of infectious TB patients by interrogating both the index case and the named contact person on the actual duration and proximity of the contact. However, these personal and travel costs per patient are highly variable and a fixed example value is almost impossible to define. Also, it was not possible to consider the costs incurred in arranging and funding local interpreters for the wide range of different languages of the mostly foreign-born patients for which no reliable estimates can be made. As such, our updated analysis represents - as in the original analysis - only the minimum costs that may be incurred in Germany for diagnosing and treating a non-MDR-TB case in Germany and for performing the necessary contact investigations.

\section{Conclusion}

Considering the inflation rate since 2011, the total cost per non-MDR-TB patient in 2019 remains stable from a societal perspective as increasing treatment costs have been fully compensated by a considerably lower loss of productivity per patient and lower public health costs. Nevertheless, because the absolute number of TB cases has clearly increased in the course of the 2015 immigrant crisis, TB is still a disease of significant economic impact in Germany.

Acknowledgements: Many thanks to Walter Haas and Bonita Brodhun (Robert Koch Institute, Berlin, Germany) who provided very helpful special evaluations of epidemiological surveillance data.

Conflict of interest: R. Diel reports a grant from Stiftung Oskar-Helene-Heim, Berlin, Germany, during the conduct of the study. A. Nienhaus has nothing to disclose.

Support statement: This work was supported by a grant from the Stiftung Oskar-Helene-Heim, Berlin, Germany. Funding information for this article has been deposited with the Crossref Funder Registry.

\section{References}

World Health Organization. Global Tuberculosis Report 2019. Geneva, WHO, 2019.

Diel R, Rutz S, Castell S, et al. Tuberculosis: cost of illness in Germany. Eur Respir J 2012; 40: 143-151.

Diel R, Vandeputte J, de Vries G, et al. Costs of tuberculosis disease in the European Union: a systematic analysis and cost calculation. Eur Respir J 2014; 43: 554-565.

4 Robert Koch Institute. Bericht zur Epidemiologie der Tuberkulose in 2009. Robert Koch Institute, Berlin, 2011.

5 Robert Koch Institute. Bericht zur Epidemiologie der Tuberkulose in 2018. Robert Koch Institute, Berlin, 2019. 
6 Diel R, Nienhaus A, Lampenius N, et al. Cost of multi drug resistance tuberculosis in Germany. Respir Med 2014; 108: 1677-1687.

7 Schaberg T, Bauer T, Brinkmann F, et al. [Tuberculosis Guideline for Adults - Guideline for Diagnosis and Treatment of Tuberculosis including LTBI Testing and Treatment of the German Central Committee (DZK) and the German Respiratory Society (DGP)]. Pneumologie 2017; 71: 325-397.

8 Federal Association of Statutory Health Insurance Physicians. Physicians Fee Schedule. http://k bv.de/html/ebm. php. Date last accessed: December 17, 2019.

9 InEK - Institut für das Entgeltsystem im Krankenhaus. Definitionshandbuch 2019; G-DRG-Version 2019. InEK, Siegburg, Germany, 2019.

10 InEK - Institut für das Entgeltsystem im Krankenhaus. Datenveröffentlichung gem. $\$ 21$ KHEntgG. https:// browser.inek.org. Date last accessed: 17 January 2020

11 Graf von der Schulenburg JM, Greiner W, Jost F, et al. German recommendations on health economic evaluation: third and updated version of the Hanover Consensus. Value Health 2008; 11: 539-544.

12 Statistisches Bundesamt. Volkswirtschaftliche Gesamtrechnungen; Fachserie 18, Reihe 1.2 2. Vierteljahr 2019, 2.15 Arbeitnehmerentgelt je Arbeitnehmer. Wiesbaden; August 2019.

13 Statistisches Bundesamt. Ergebnisse des Mikrozensus 2019; Fachserie 1 - Bevölkerung und Erwerbstätigkeit, Reihe 4.1. Wiesbaden; August 2019.

14 Diel R, Loytved G, Nienhaus A, et al. [New recommendations for contact tracing in tuberculosis]. Gesundheitswesen 2011; 73: 369-388.

15 Diel R, Nienhaus A, Lange C, et al. Cost-optimisation of screening for latent tuberculosis in close contacts. Eur Respir J 2006; 28: 35-44.

16 de Vries G, Baltussen R. Kosten van tuberculose en TBC-bestrijding in Nederland [Cost of tuberculosis and tuberculosis control in the Netherlands]. Tegen Tuber 2013; 109: 3-7.

17 Floe A, Hilberg O, Wejse C, et al. The economic burden of tuberculosis in Denmark 1998-2010. Cost analysis in patients and their spouses. Int J Infect Dis 2015; 32: 183-190.

18 Gullón JA, Garcia-Garcia JM, Villanueva MA, et al. Tuberculosis costs in Spain and related factors. Arch Bronconeumol 2016; 52: 583-589. 